\title{
Validation of a stochastic droplet breakup model applied to a liquid jet in turbulent crossflow
}

\author{
R. Blanchard ${ }^{1} \& \mathrm{~S} . \mathrm{Shi}^{2}$ \\ ${ }^{I}$ GE Global Research, USA \\ ${ }^{2}$ GE Power \& Water, USA
}

\begin{abstract}
Coal gasification technology has the potential to reduce the environmental impact of coal power by enabling technologies like integrated gasification combined cycle (IGCC) and carbon capture for sequestration (CCS) for power generation. Numerical simulations of the various components of a coal gasification plant using validated CFD models enables faster configuration improvement cycles and thereby increased component performance, reliability, and overall cycle efficiency. Many such computations require the simulation of turbulent, multi-phase flows where the atomization and agglomeration of liquids play a critical role in plant processes. This paper focuses on the development and validation of a stochastic droplet breakup and agglomeration model for use in a steady-state RANS simulation of a liquid jet in a turbulent cross-flow. The models aim to accurately predict the trajectories and sizes of liquid droplets without incurring the computational cost of more expensive methodologies such as LES or VOF which are currently too computationally intensive to be used in the design cycle. The model builds on similar models proposed by Apte and Kuan by proposing a new probability density function (PDF) for the break-up process and adapting the methodology to a steady-state framework. The model is then validated against measurements made by $\mathrm{Wu}$. The model shows good qualitative and quantitative agreement with measurement of the downstream mass flux distribution of the liquid droplets. An agglomeration model is added to the simulation which improves the agreement between predicted and measured Sauter-mean diameter (SMD) of the liquid droplets downstream of the initial atomization region.
\end{abstract}

Keywords: atomization, agglomeration, stochastic, gasification. 


\section{Introduction}

Coal-derived power plays a major role in meeting the world's energy needs and will continue to do so for many years. Technologies can make coal-derived power generation with fewer emissions than traditional coal plants. Coal gasification technology has the potential to reduce the environmental impact of coal power by enabling technologies like integrated gasification combined cycle (IGCC and carbon capture for sequestration (CCS) for power generation. Numerical simulations of the various components of a coal gasification plant using validated CFD models enables faster configuration improvement cycles and thereby provide increased component performance, reliability, and overall cycle efficiency. Many such computations require the simulation of turbulent, multi-phase flows where the atomization and agglomeration of liquids play a critical role in plant processes.

This paper focuses on the development and validation of a stochastic droplet breakup and agglomeration model for use in a steady-state RANS simulation of a liquid jet in a turbulent cross-flow. The goal of the current study is to accurately predict the trajectories and sizes of the atomized particles using a discrete phase model (DPM) coupled with a 2-equation turbulence model without incurring the computational cost of more expensive methodologies such as large eddy simulation (LES) or volume of fluid (VOF) which are currently too computationally intensive to be used in the design cycle.

\section{Breakup model formulation}

The proposed breakup model is similar to the previously-published models by Apte [1] and Kuan [2] in that breakup events are triggered when a droplet's Weber number exceeds a critical value (this work assumes that $\mathrm{We}_{\mathrm{CR}}=6$ ) and the and the size of the child droplets are determined by a stochastic sampling of a probability density function (PDF) based on Weber number. The current model differs from previous versions in 3 key areas:

1. The breakup PDF formulation prevents particle growth (child droplets cannot be larger than their parents).

2. The model is implemented for steady-state spray simulations.

3. Droplet parcelization is held constant throughout the atomization process.

\subsection{Breakup PDF}

The proposed breakup function from which new particle diameters are sampled, exhibits many of the same self-similar properties described by Apte [1] but is formulated such that no child droplet can be larger than its parent. The function is formulated such that the ratio of droplet Weber number to critical Weber number is the key parameter that drives the rate of breakup during the atomization process and is shown in cumulative distribution function (CDF) form in equation (1), where $\mathrm{i}$ and $\mathrm{i}+1$ subscripts respectively denote parent and 
child droplet characteristics. The timescale of droplet breakup is given in equation (2), where $\rho \mathrm{p}, \rho \mathrm{g}$, dp, and Vp-g denote droplet density, gas density, droplet diameter, and the magnitude of relative velocity between the droplet and gas respectively. No further breakup events are allowed to occur until during the time interval $\Delta \mathrm{tBU}$ after a breakup event is triggered. The value of the constant $\mathrm{C}$ in equation (2), is taken to be $\sqrt{1 / 3} \sqrt{1 / 3}$ from O'Rourke [3]. The definition of Weber number is given in (3), where $\sigma$ denotes droplet surface tension.

$$
\begin{gathered}
C D F=p_{o}\left(\frac{W e_{i+1}}{W e_{i}}\right)\left(\frac{W e_{i}}{W e_{C R}}\right) \\
\Delta t_{B U}=C \sqrt{\frac{\rho_{p}}{\rho_{g}}} \frac{d_{p}}{V_{p-g}} \\
W e=\frac{\rho_{p} V_{p-g}^{2} d_{p}}{\sigma_{g}}
\end{gathered}
$$

\subsection{Numerical implementation and parcelization}

The droplet breakup model algorithm is implemented in the following steps:

1. A new particle parcel stream is introduced into the simulation with diameter equal to the injector orifice diameter.

2. As the particle is marched through the steady-state flow field, the particle's Weber number is calculated at each time step, if the Weber number is ever greater than $\mathrm{We}_{\mathrm{CR}}$ and the elapsed time since the last breakup event (or injection) is greater than $\Delta t_{\mathrm{BU}}$, a breakup event is triggered.

When a breakup event is triggered and a new particle diameter is sampled from equation (1) but the parcelization of the stream is held constant as the new particle diameter is applied to the entire parcel stream.

\section{Validation}

The current model was applied to a simulation of an experiment by $\mathrm{Wu}$ et al. $[4,5]$, which consisted of a liquid jet injected into a turbulent crossflow of gas at low Mach number. Measurements of mass flux, particle velocity, and particle size were made along the spray centerline 300 nozzle diameters downstream of the injection point.

The CFD simulation of the experimental test section was modelled as shown in as a $12.5 \times 40.6 \times 7.5 \mathrm{~cm}$ rectangular domain with $1.0 \mathrm{e} 6$ grid cells and wall $\mathrm{y}+$ 
of approximately 20 for the baseline simulation. The baseline conditions consisted of a $.5 \mathrm{~mm}$ diameter liquid jet (all distance normalization of the results is done based on this diameter) being injected at $19.3 \mathrm{~m} / \mathrm{s}$ into a $103 \mathrm{~m} / \mathrm{s}$ gas crossflow. Turbulence was modelled using the standard k-epsilon model and droplet-turbulence interaction was accounted for using Fluent's default stochastic random walk model. The spray was parcelized into $1 \mathrm{e} 5$ streams of equal mass flow. The liquid jet of pure water, was assumed to have surface tension and viscosity of $7.19 \mathrm{e}-2 \mathrm{~N} / \mathrm{m} 1.003 \mathrm{e}-3 \mathrm{~Pa}-\mathrm{s}$ respectively. The gas flow was assumed to have properties of air at standard sea-level conditions.

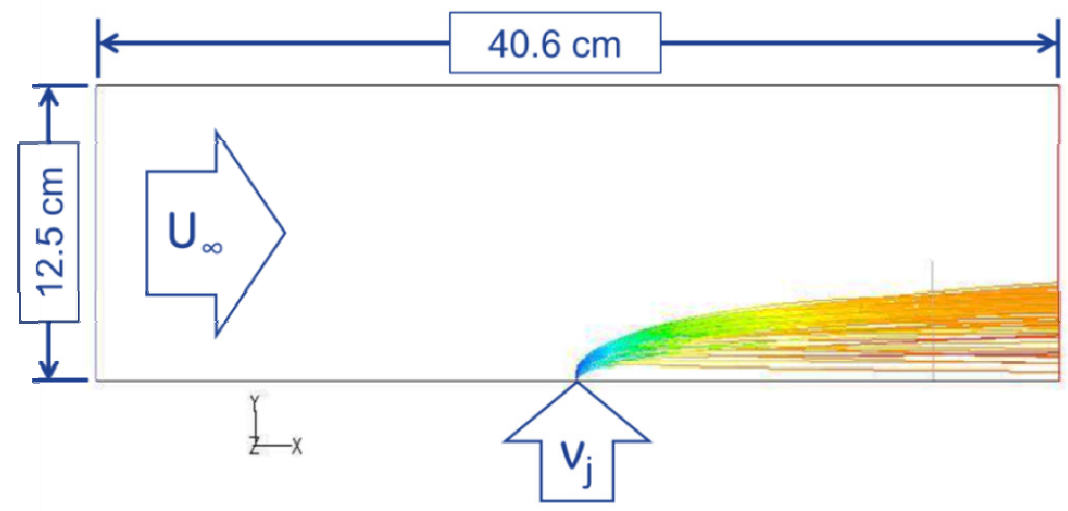

Figure 1: $\quad$ Schematic of wind tunnel and liquid jet.

In addition to the baseline case, case A, four additional runs were completed, shown in, to evaluate the sensitivity of the results to changes in liquid jet velocity (at 12.3 and $29 \mathrm{~m} / \mathrm{s}$ respectively) and changes in crossflow velocity (69 and $137 \mathrm{~m} / \mathrm{s}$ respectively). The mass flux profiles of these streams were also sampled along the spray centerline 300 injector diameters downstream of the liquid jet.

Table 1: $\quad 5$ cases comprising this study.

\begin{tabular}{|c|c|c|}
\hline Case & $\mathrm{U}(\mathrm{m} / \mathrm{s})$ & $\mathrm{v}_{\mathrm{j}}(\mathrm{m} / \mathrm{s})$ \\
\hline$A$ & 103 & 19.3 \\
\hline$B$ & 103 & 12.8 \\
\hline$C$ & 103 & 29 \\
\hline$D$ & 69 & 19.3 \\
\hline$E$ & 137 & 19.3 \\
\hline
\end{tabular}


The droplet flux of the simulated spray showed good first- and secondmoment agreement with the measured flux profiles as can be seen in Figure 2. While certainly some discrepancy exists between the vertical location of the measured and simulated peaks in the flux profiles, the centroids of the profiles match each other within 3-10\% in all five cases as shown in Figure 3. The halfwidths of the profiles are likewise similar between simulation and measurement.
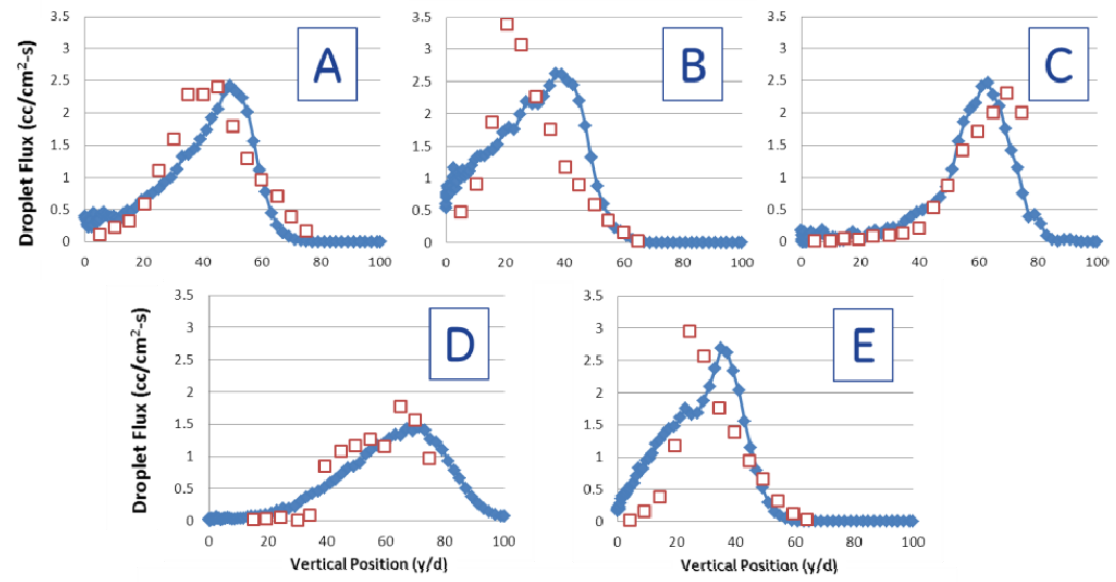

- This Study

Wu (1997)

Figure 2: Droplet flux profiles at $\mathrm{x} / \mathrm{d}=300$ downstream of the injection point.

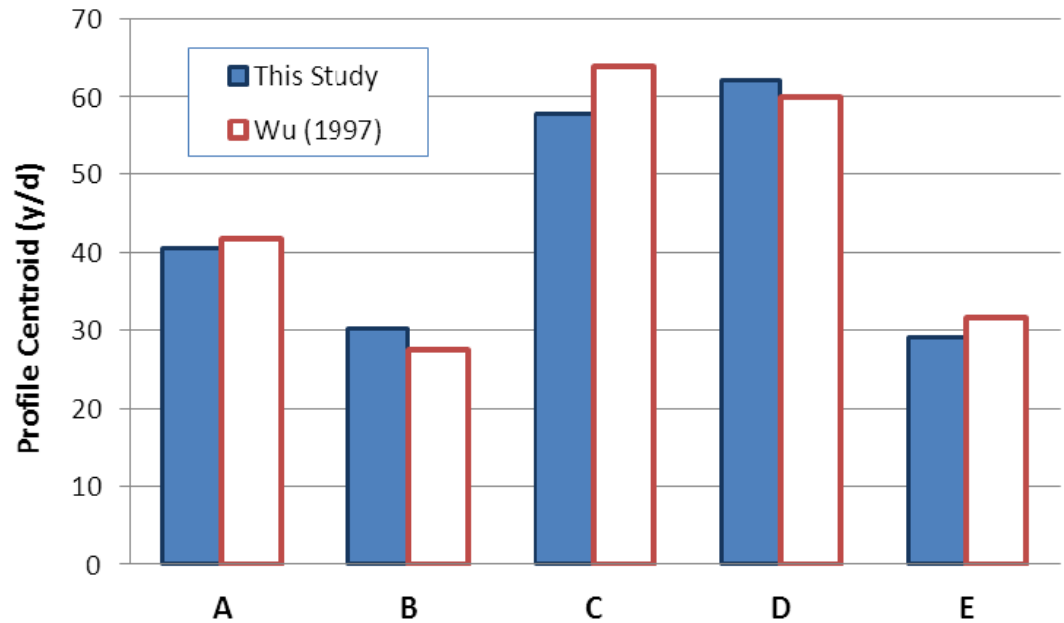

Figure 3: Comparison of profile centroids between the current study and measurements. 
Table 2: Comparison of profile centroids between current study and measurements.

\begin{tabular}{|c|c|c|c|c|}
\hline & & & \multicolumn{2}{|c|}{ Centroid Height (y/d) } \\
\hline Case & $\mathrm{U}(\mathrm{m} / \mathrm{s})$ & $\mathrm{v}_{\mathrm{j}}(\mathrm{m} / \mathrm{s})$ & Current Work & $\mathrm{Wu}(1997)$ \\
\hline $\mathrm{A}$ & 103 & 19.3 & 40.6 & 41.9 \\
\hline $\mathrm{B}$ & 103 & 12.8 & 30.1 & 27.5 \\
\hline $\mathrm{C}$ & 103 & 29 & 57.9 & 63.8 \\
\hline $\mathrm{D}$ & 69 & 19.3 & 62.1 & 60.0 \\
\hline $\mathrm{E}$ & 137 & 19.3 & 29.0 & 31.5 \\
\hline
\end{tabular}

\section{Agglomeration model}

While the simulated particle trajectories showed good agreement with the measured profiles of particle mass flux, the profiles of Sauter-mean diameter (SMD) of the simulated sprays showed consistently smaller droplet sizes than were measured. It was hypothesized that the droplets were breaking and very rapidly reaching their final height above the injection plane but were coalescing to form larger droplets farther downstream before being measured. This hypothesis, if true, would explain how a droplet's height at the measurement plane could be decoupled somewhat from its size.

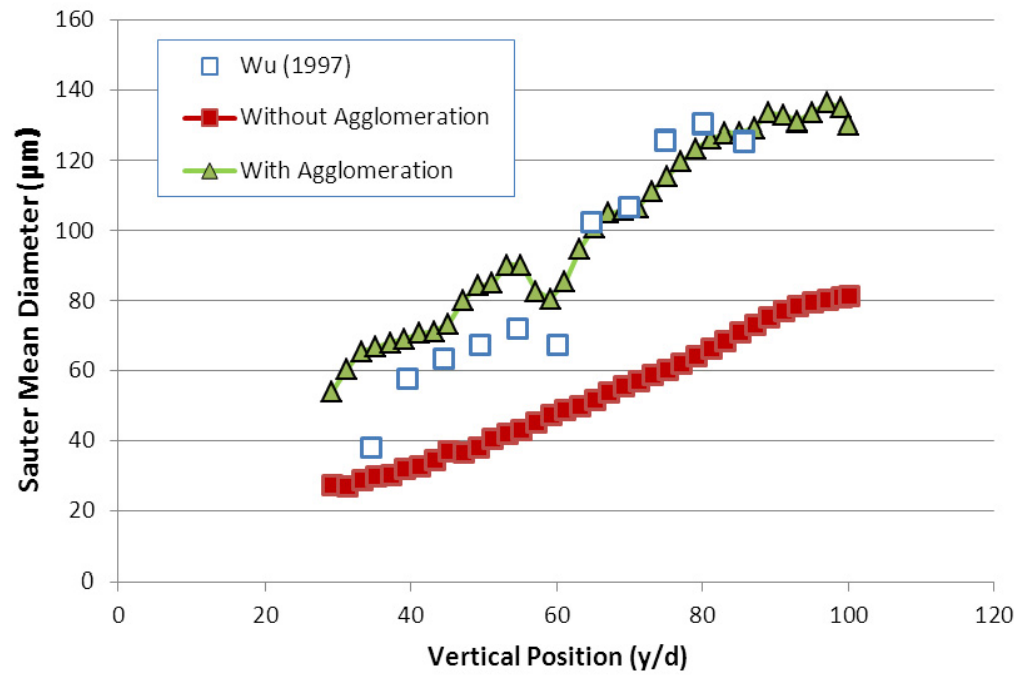

Figure 4: $\quad$ Sauter-mean diameter profiles at $\mathrm{x} / \mathrm{d}=300$. 
To account for agglomeration of droplets a simple droplet collision and coalescence model, originally developed by O'Rourke [3] for unsteady sprays, was adapted to a steady-state formulation as shown in equations (4) and (5), where $\mathrm{K}_{\mathrm{T}} \Delta \mathrm{t}_{\mathrm{p}} \mathrm{m}_{\mathrm{p}}, \mathrm{C}_{\mathrm{DPM}}$, and smd denote, local turbulent kinetic energy, droplet trajectory integration timestep, parcel mass, local droplet concentration, and the local SMD of the spray respectively. The effect of this model on the size distribution of the simulated spray is shown in Figure 4, which shows how the inclusion of the agglomeration model significantly improves the agreement between measured and simulated SMD profiles.

$$
\begin{gathered}
V=\frac{3}{2} \sqrt{K_{T}} \Delta t_{p}\left(\frac{m_{p}}{d_{p}}+\frac{C_{D P M}}{s m d}\right) \\
\frac{d_{i+1}}{d_{i}}=\sqrt[3]{1+V}
\end{gathered}
$$

\section{Conclusions}

The proposed steady state breakup model showed good agreement with measurements of droplet flux profiles downstream of the injector. When a simple droplet agglomeration model was incorporated into the simulation, the size distributions of the simulated spray matched the corresponding measurements as well. This simple set of models allows for the atomization process to be modelled with reasonable accuracy in a steady state framework that significantly reduces calculation time compared to other, more computationally intensive methods like volume-of-fluid or large eddy simulation, thus providing the designer with a low-cost option for simulating jet breakup.

(C) 2011 General Electric Company. All Rights Reserved.

This material may not be copied or distributed in whole or in part, without prior permission of the copyright owner and the publisher.

\section{References}

[1] Apte S.V., Gorokhovski, M., Moin, P., LES of atomizing spray with stochastic modeling of secondary breakup, International Journal of Multiphase Flow. 29, pp. 1503-22, 2003.

[2] Kuan, B., CFD Modelling of Liquid Jet and Cascade Breakup in Crossflows, Seventh Seventh International Conference on CFD in the Minerals and Process Industries, CSIRO: Melbourne, Australia, 2009.

[3] O'Rourke, P.J., Collective Drop Effects on Vaporizing Liquid Sprays, $P h D$ Thesis, Princeton University: Princeton, New Jersey, 1981. 
[4] Wu, P.K., Kirkendell, K.A., Fuller, R.P., "Spray Structures of Liquid Jets Atomized in Subsonic Crossflows" Journal of Propulsion and Power, 13(2), pp. 64-73, 1997.

[5] Wu, P.K., Kirkendell, K.A., Fuller, R.P., Spray Structures of Liquid Jets Atomized in Subsonic Crossflows. Journal of Propulsion and Power, 14(21), pp. 173-182 1998. 